\title{
Pressure-induced changes in the structural and absorption properties of crystalline 5-nitramino-3,4-dinitropyrazole
}

\author{
DONG XIANG, QIONG WU, ZHICHAO LIU, WEIHUA ZHU* and HEMING XIAO \\ Institute for Computation in Molecular and Materials Science and Department of Chemistry, Nanjing \\ University of Science and Technology, Nanjing 210094, China \\ e-mail: zhuwh@njust.edu.cn
}

MS received 30 May 2015; revised 3 July 2015; accepted 15 July 2015

\begin{abstract}
Periodic density functional theory with dispersion correction (DFT-D) was used to study the structural, electronic, and absorption properties of crystalline 5-nitramino-3, 4-dinitropyrazole (NADNP) under hydrostatic compression of 0-140 GPa. The results indicate that the PBE-G06 is the best functional for studying NADNP. As the pressure increases, the lattice of parameters, band gap, density of states and absorption spectra change regularly except for $126 \mathrm{GPa}$, where NADNP begins to decompose and form a new bond. An analysis of the band gap and density of states indicates that NADNP becomes more and more sensitive under compression. The absorption spectra show that NADNP has relatively high optical activity with increasing pressure.
\end{abstract}

Keywords. DFT with dispersion-correction (DFT-D); 5-nitramino-3, 4-dinitropyrazole; hydrostatic pressure; structures; optical absorption

\section{Introduction}

Nitraminopyrazoles, ternary hydrogen-bond induced high-energy density materials, feature strong interand intramolecular interactions between nitro, amino, and nitramine that lead to high density, excellent performance, and good stability. It is reported that 5nitramino-3,4-dinitropyrazole (NADNP) (figure 1) has a remarkable measured density of $1.97 \mathrm{~g} \mathrm{~cm}^{-3}$ at 298 $\mathrm{K}$, which is consistent with its crystal density $(2.032$ $\mathrm{g} \mathrm{cm}^{-3}, 150 \mathrm{~K}$ ), and ranks highest among azole-based CHNO compounds. ${ }^{1}$ In addition, the ternary hydrogenbonds in NADNP result in their acceptable impact and friction sensitivities. So, NADNP is chosen as a model substrate. Other highly energetic compounds that have high densities, excellent performance and good stability could be used in similar studies.

It is known that the information on the energetic compounds under extreme conditions is helpful for their safe use and cost-effective disposal. Thus, it is necessary to study the behavior of NADNP under high pressures. To study the pressure effects in energetic compounds is a challenging experimental task. An alternative approach is the atomistic simulation, an effective way to model the physical and chemical properties of complex solids at the atomic level as a complement to experimental work. Density functional theory (DFT) method with pseudopotentials and a plane-wave basis

\footnotetext{
*For correspondence
}

set has been successfully applied to study the structures and properties of energetic solids under hydrostatic compressions..$^{2-15}$

In this study, periodic DFT with dispersioncorrection (DFT-D) was used to study the structure and absorption properties of NADNP under hydrostatic pressure of $0-140 \mathrm{GPa}$. The atomic positions and the unit cell parameters were allowed to relax to the minimum energy configuration to investigate the crystal structure at different pressures. Then, we examined the variations in electronic structure under compression. Finally, we compared their optical absorption properties.

\section{Computational method}

The calculations performed in this study were done within the framework of DFT ${ }^{16}$ as implemented in the Cambridge Serial Total Energy Package (CASTEP) code. ${ }^{17}$ The calculations were done using Vanderbilt ultrasoft pseudopotentials for all atoms ${ }^{18}$ and generalized gradient approximation ${ }^{19}$ (GGA) with $\mathrm{PBE}^{20}$ (Perdew Burke Ernzerhof) and PW91 ${ }^{21}$ (Perdew and Wang) functionals. To correct DFT for missing vdW interactions, we used three types of corrections such as the Grimme ${ }^{22}$ (G06), the Tkatchenko and Scheffler $(\mathrm{TS})^{23}$ corrections to the PBE, and the Ortmann, Bechstedt, and Schmidt (OBS) ${ }^{24}$ correction to the PW91. ${ }^{25}$ The self-consistent ground state of the system was 


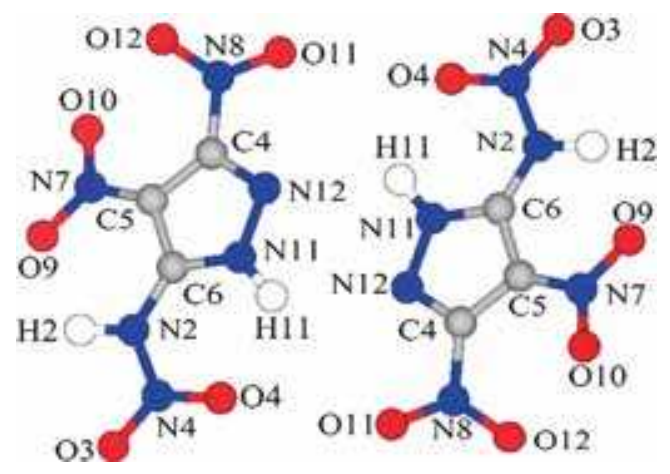

Figure 1. Crystal structure and atomic numbering of NADNP.

determined by using a band-by-band conjugate gradient technique to minimize the total energy of the system with respect to the plane-wave coefficients. The electronic wave functions were obtained by a densitymixing minimization method ${ }^{26}$ and the structures were relaxed by using the Broyden, Fletcher, Goldfard, and Shannon (BFGS) method. ${ }^{27}$ Geometry optimization is based on reducing the magnitude of calculated forces and stresses until they become smaller than defined convergence tolerances. Therefore, it is possible to specify an external stress tensor to model the behavior of the system under tension, compression, shear, etc. In these cases, internal stress tensor is iterated until it becomes equal to the applied external stress. The cutoff energy of plane waves was set to $340.0 \mathrm{eV}$. Brillouin zone sampling was performed by using the MonkhostPack scheme with a $k$-point grid of $2 \times 1 \times 1$. The values of the kinetic energy cutoff and the $k$-point grid were determined to ensure the convergence of total energy.

To compare with experiments, we used the crystal structure of NADNP at ambient pressure and temperature as input structure. The NADNP crystallizes in an orthorhombic lattice with $P-1$ space group and contains four $\mathrm{C}_{3} \mathrm{~N}_{6} \mathrm{O}_{6} \mathrm{H}_{2}$ molecules per unit cell. ${ }^{1}$ Figure 1 displays crystal and atomic structure of NADNP. The experimental crystal structure of NADNP was relaxed to allow the ionic configurations, cell shape, and volume to change at zero pressure. Then, from the relaxed structure, we applied hydrostatic compression of 0-140 $\mathrm{GPa}$. All the calculations are based on the same crystal structure of NADNP. In the geometry relaxation, the total energy of the system was converged to less than $1.0 \times 10^{-5} \mathrm{eV}$, the residual force less than $0.03 \mathrm{eV} / \AA$, the displacement of atoms less than $0.001 \AA$, and the residual bulk stress less than $0.05 \mathrm{GPa}$ for all the equilibrium structures.

\section{Results and Discussion}

Before carrying out the calculations reported here, we applied three different functionals (PBE-TS, PBE-OBS, PW91-OBS) to relax the bulk NADNP at ambient pressure without any constraint. Table 1 lists the experimental and relaxed cell parameters of NADNP. The lattice parameters estimated by the PBE-G06 here is much closer to the experimental value than those by the PW91-OBS and PBE-TS. In addition, the results calculated by the PW91-OBS and PBE-TS are obviously different from the experimental values, suggesting that the PW91-OBS and PBE-TS is not suitable for investigating the NADNP crystal. Table 2 presents the PBE-G06calculated bond lengths and bond angles of NADNP along with the experimental data. It is seen that the calculated bond lengths and bond angles agree with the experimental values. Thus, we adopted the PBE with the G06 correction to study the structure and properties of NADNP under high pressure here.

\subsection{Crystal structure}

Figure 2 displays the relaxed lattice constant $(a, b, c)$ of NADNP in the pressure range of 0-140 GPa. Overall, $a, b$, and $c$ decrease with the increasing pressure. But the pressure-induced change trends of the lattice constants are different. It is seen in figure 2 that the $b$ axis

Table 1. Comparison of relaxed lattice parameters of NADNP with experimental data at ambient conditions.

\begin{tabular}{lccccccc}
\hline Method & $a(\AA)$ & $b(\AA)$ & $c(\AA)$ & $\alpha$ & $\beta$ & $\gamma$ & Cell volume $\left(\AA^{3}\right)$ \\
\hline Expt. & 6.4598 & 9.7023 & 11.5825 & 100.725 & 90.314 & 91.109 & 713.087 \\
PW91-OBS & 6.74848 & 10.4076 & 12.1076 & 103.042 & 90.8188 & 90.7245 & 828.247 \\
& $(-4.47)$ & $(-7.27)$ & $(-4.53)$ & $(-2.3)$ & $(-0.56)$ & $(0.42)$ & $(-16.15)$ \\
PBE-TS & 6.6474 & 10.3425 & 12.1326 & 101.991 & 90.3338 & 90.6899 & 815.388 \\
& $(-2.90)$ & $(-6.60)$ & $(-4.75)$ & $(-1.26)$ & $(-0.42)$ & $(0.46)$ & $(-14.35)$ \\
PBE-G06 & 6.65256 & 10.1282 & 11.9997 & 101.716 & 90.6647 & 90.9564 & 791.470 \\
& $(-2.98)$ & $(-4.39)$ & $(-3.60)$ & $(-0.98)$ & $(-0.39)$ & $(0.17)$ & $(-10.99)$ \\
\hline
\end{tabular}

Note. The values in parentheses correspond to the percentage differences relative to the experimental data. 
Table 2. Experimental and calculated bond lengths $(\AA)$ and angles $\left(^{\circ}\right)$ in NADNP crystal at ambient conditions.

\begin{tabular}{lccccc}
\hline Bond Lengths & PBE-G06 & Expt. & Bond Angles & PBE-G06 & Expt. \\
\hline O8-N5 & 1.256 & 1.227 & N2-C6-C5 & 127.12 & 127.05 \\
N6-C2 & 1.413 & 1.418 & C4-N8-O12 & 116.71 & 116.927 \\
N9-H9 & 1.03 & 0.843 & H11-N11-N12 & 120.273 & 120.281 \\
N1-H1 & 1.304 & 0.813 & N12-C4-C5 & 112.196 & 126.623 \\
N3-O1 & 1.256 & 1.226 & O4-N4-O3 & 126.6 & 126.462 \\
\hline
\end{tabular}

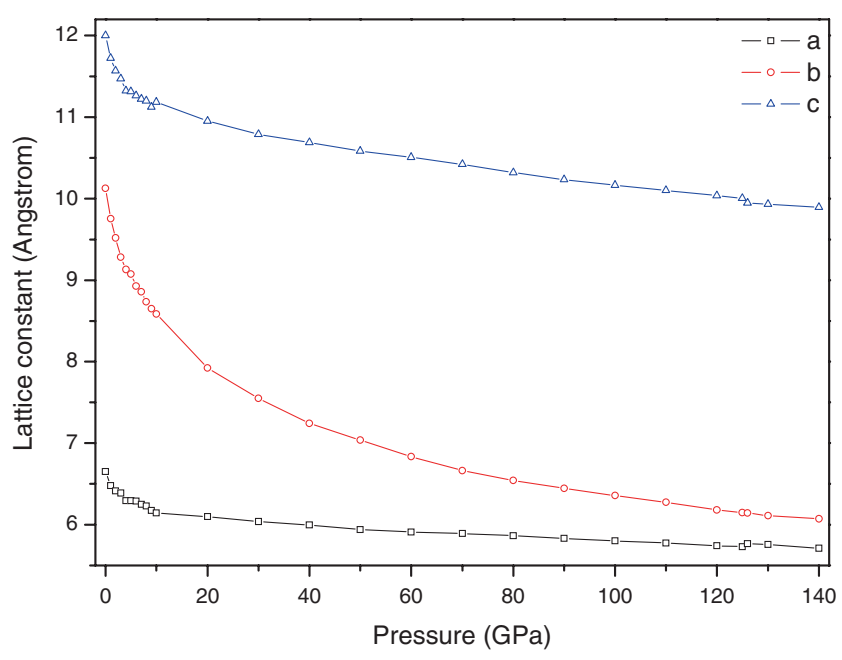

Figure 2. Lattice constants $(a, b, c)$ of NADNP in the pressure range of $0-140 \mathrm{GPa}$.

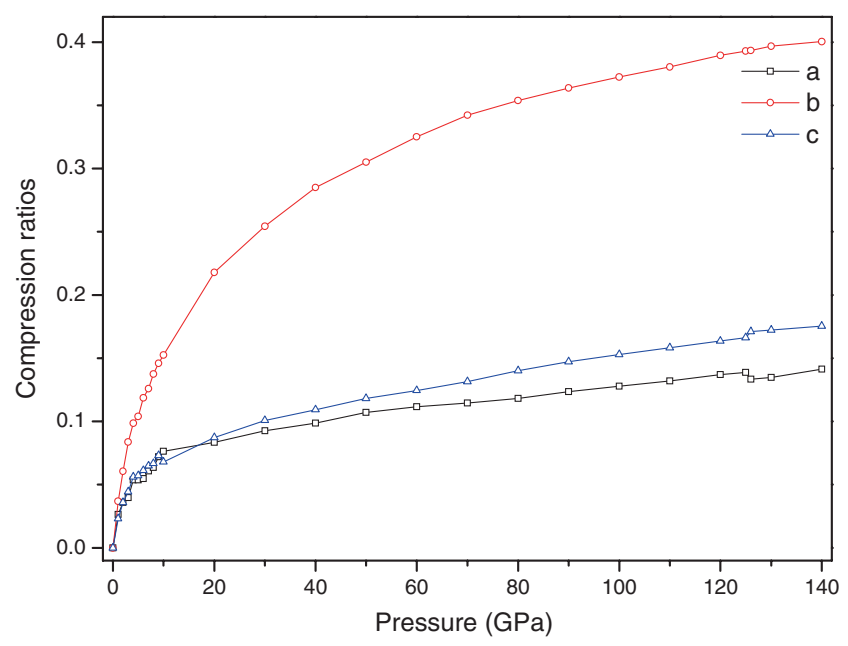

Figure 3. Lattice constant $(a, b, c)$ g compression ratio of NADNP in the pressure range of 0-140 GPa.

is the most compressible. This indicates that the compressibility of NADNP crystal is anisotropic. Figure 3 displays the relaxed lattice constant $(a, b, c)$ compression ratio of NADNP. In the pressure range of $0-140$ $\mathrm{GPa}$, the total compression ratio increases in the order of $b<c<a$, indicating that the structure is much softer in the $a$ direction than in the $b$ and $c$ axis.

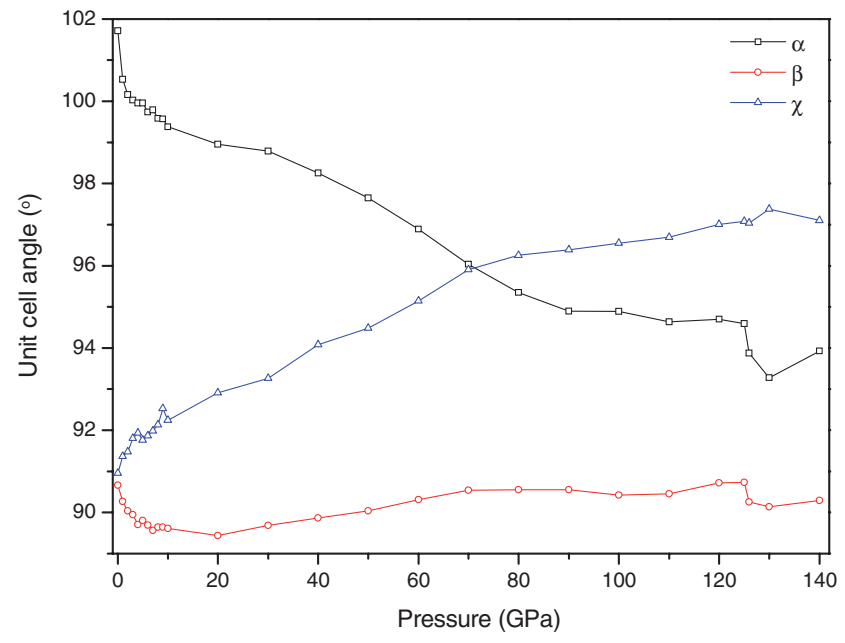

Figure 4. Lattice angles $(\alpha, \beta, \chi)$ of NADNP in the pressure range of 0-140 $\mathrm{GPa}$.

Figure 4 displays the relaxed unit cell angles $(\alpha, \beta$, $\chi$ ) of NADNP in the pressure range of 0-140 GPa. As the pressure increases, $\chi$ gradually increases; so does $\beta$ after $20 \mathrm{GPa}$, while $\alpha$ decreases. But they all undergo sudden, obvious changes at $126 \mathrm{GPa}$, which suggest that large changes in crystalline structure may take place at this pressure. The unit cell angles $\alpha$ and $\beta$ decrease suddenly, but $\chi$ increases abruptly at $126 \mathrm{GPa}$. Figure 5 displays the relaxed unit cell angles compression ratio of NADNP. In the pressure range of $0-125$ $\mathrm{GPa}$, the variation tendency of the compression ratio of $\alpha$ increases, whereas those of the $\beta$ and $\chi$ decrease. In all, the total compression ratios increase in the order $\alpha>\beta>>\chi$ in the whole pressure range, indicating that the $\beta$ and $\chi$ angles are much softer than $\alpha$. Pressureinduced changes of the unit-cell volumes are presented in figure 6. The unit cell volume decreases monotonically. At about $80 \mathrm{GPa}$, the volume compression is up to $50 \%$.

\subsection{Molecular Structure}

The applied pressure causes the changes not only in the unit cell of NADNP but also in its molecular geometry like the bond lengths and bond angles. Figure 7 


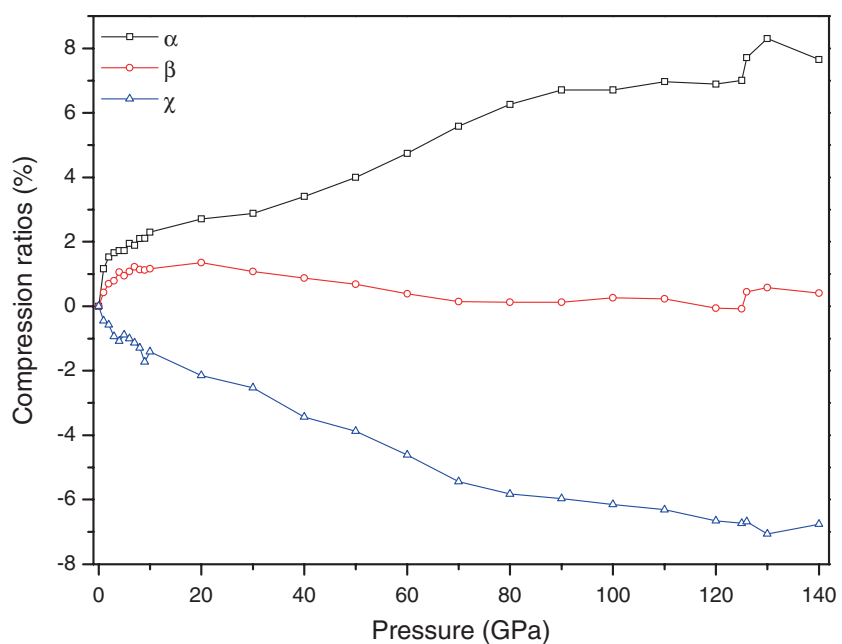

Figure 5. Compression ratio of NADNP in the pressure range of 0-140 GPa.

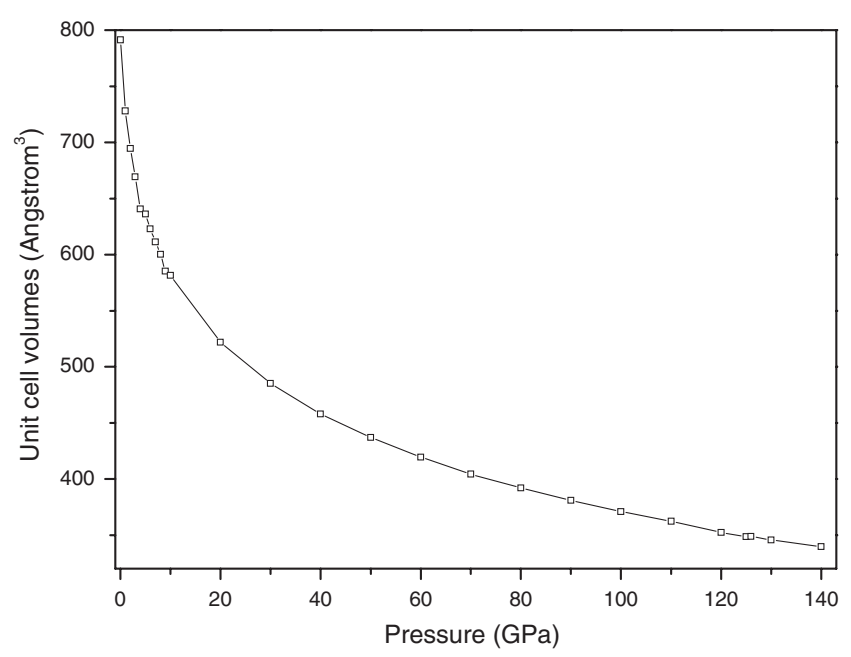

Figure 6. Unit cell volumes of NADNP in the pressure range of 0-140 GPa.

presents the selected five bond angles (O11-N8-O12, C4-C5-C6, N12-N11-H11, C6-N2-H2, and O4-N4-N2 angles) of NADNP at different pressures. In the range of 0-125 GPa, all the angles decrease monotonically. At $126 \mathrm{GPa}$, the O11-N8-O12 and N12-N11-H11 angles decrease suddenly, while the C6-N2-H2, O4-N4-N2, and $\mathrm{C} 4-\mathrm{C} 5-\mathrm{C} 6$ angles increase abruptly. In the range of 126-140 GPa, the O11-N8-O12, C6-N2-H2, O4N4-N2, and C4-C5-C6 angles decrease monotonically, whereas the N12-N11-H11 angle increases monotonically. This indicates that the structure is changed at the pressure of $126 \mathrm{GPa}$.

Figure 8 presents the atom distance between N12' and $\mathrm{H} 11$ in NADNP at different pressures. When the pressure increases from 0 to $125 \mathrm{GPa}$, the atomic distance between N12' and H11 decreases as a whole but increases in the pressure range of 126-140 GPa. At

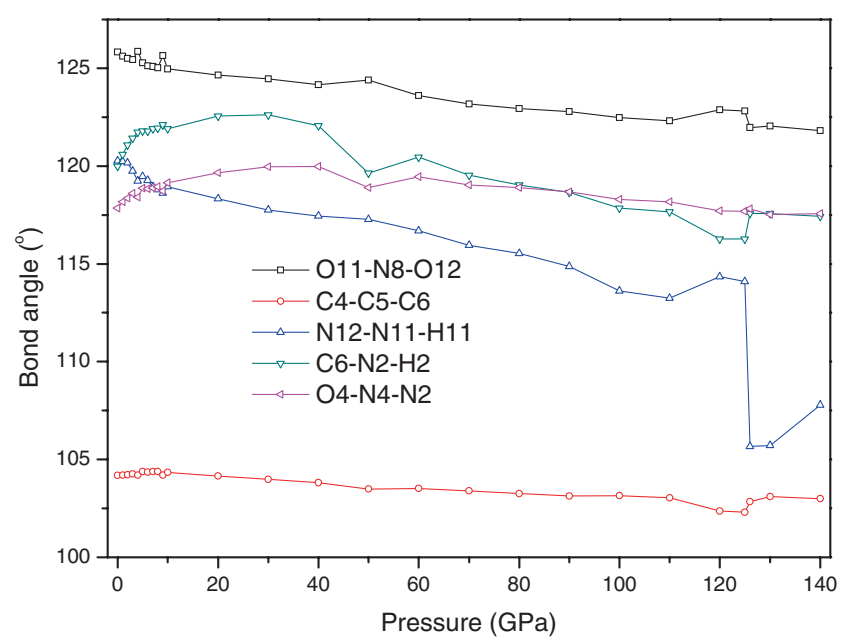

Figure 7. Bond angles of NADNP in the pressure range of 0-140 GPa.

$126 \mathrm{GPa}$, the distance between $\mathrm{N} 12$ ' and $\mathrm{H} 11$ atoms decreases abruptly from 1.738 to $1.107 \AA$, which shows that N12'and $\mathrm{H} 11$ atoms form a new bond at this pressure; moreover, the N12' and $\mathrm{H} 11$ atoms are sensitive to compression. It may thus be inferred that the formation of the H11-N12' bond may possibly trigger the NADNP decomposition.

Figure 9 displays the perspective view of the unit cell of NADNP at $0 \mathrm{GPa}$ and $126 \mathrm{GPa}$. At $0 \mathrm{GPa}$, the arrangement of the molecules in the crystalline NADNP is irregular. It is unexpected that when the pressure increases to $126 \mathrm{GPa}$, the distance between $\mathrm{N} 12$ and H11' (N12' and H11) atoms decreases from 2.542 to $1.266 \AA$, indicating that new covalent bonds between N12-H11' and N12'- H11 are formed. It can also be seen that the four molecules arrange in two regular layers in the unit cell. These obvious changes suggest that the position for each molecule in the crystal was changed at $126 \mathrm{GPa}$.

\subsection{Electronic Structure}

Band gap is an important parameter to characterize the electronic structure of solids. Figure 10 displays the band gaps of NADNP at different pressures. The band gap of NADNP gradually decreases with the increase of pressure in 0-100 GPa range, but it increases slightly in 100-125 GPa. At $126 \mathrm{GPa}$, the significant reduction of the band gap is caused by the structural change with the formation of the covalent bonds. It decreases slightly in 126-40 GPa range. The smaller the band gap, easier is the electron transfer from the valence band to the conduction band, and higher the probability that the energetic materials become decomposed and explode. , $, 10,12^{2}$ Therefore, it may be inferred that the impact sensitivity 


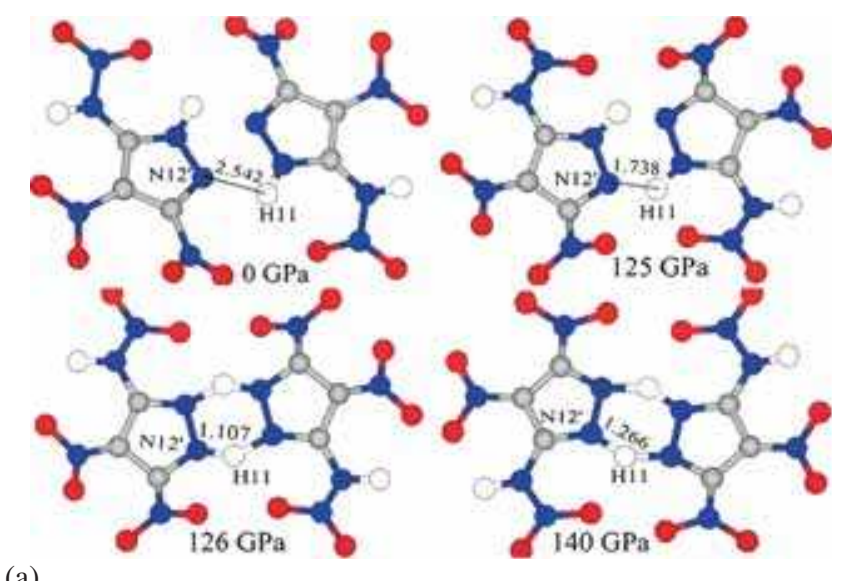

(a)

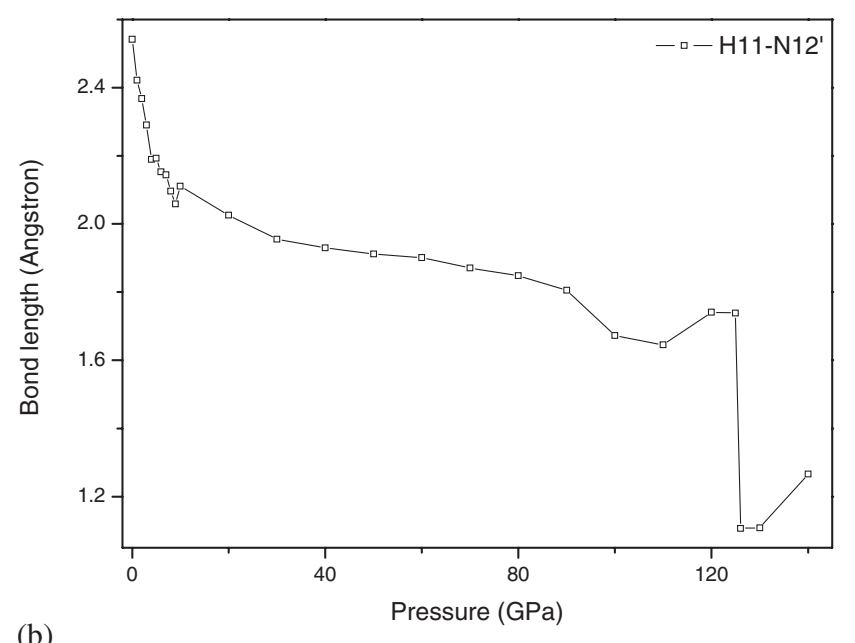

Figure 8. (a) Formation of new N12'-H11 bond, and (b) Variation of N12'-H11 bond length in NADNP at different pressures.

for NADNP becomes more and more sensitive with the increase of pressure.

In order to understand the band nature and electronic structure of the crystal NADNP at different pressures, we investigated its total density of states (DOS) and partial DOS (PDOS). Figure 11 displays the total and

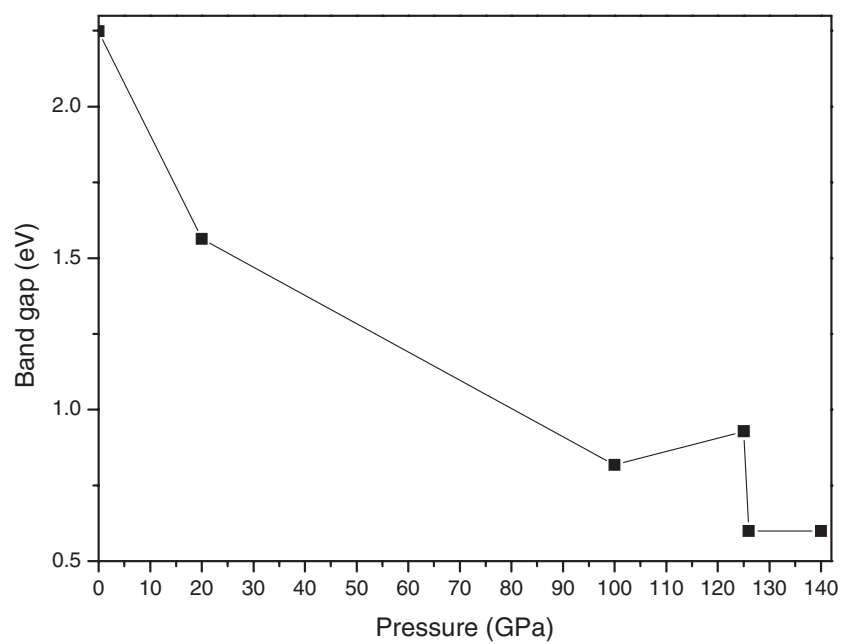

Figure 10. Band gap of NADNP as a function of hydrostatic pressure.

partial DOS of the $\mathrm{C}, \mathrm{N}$ states, $\mathrm{O}$ and $\mathrm{H}$ states of NADNP at $0 \mathrm{GPa}$. The PDOS of the $\mathrm{O}$ states and $\mathrm{N}$ states are larger than those of the $\mathrm{H}$ states and $\mathrm{C}$ states. It may be expected that the former makes more important contributions to the valence bands than the latter, indicating that the $\mathrm{O}$ states and $\mathrm{N}$ states act as active centers.

Figure 12 presents the total density of states (DOS) and partial DOS (PDOS) of NADNP at 0, 20, 125, 126 and $140 \mathrm{GPa}$. It is seen that the peaks become more and more wide and dispersed and have a tendency of shifting to lower energy with increasing pressure. This indicates that the band formation and band dispersion increase is accompanied by a broadening of DOS because the intermolecular distances are reduced and thus the intermolecular interactions are enhanced with the increasing pressure. Upon compression the DOS peaks in the conduction bands have a tendency of shifting to lower energy. Therefore, the electronic delocalization in bulk NADNP increases. At $126 \mathrm{GPa}$, the DOS peaks connect together near the Fermi energy level,
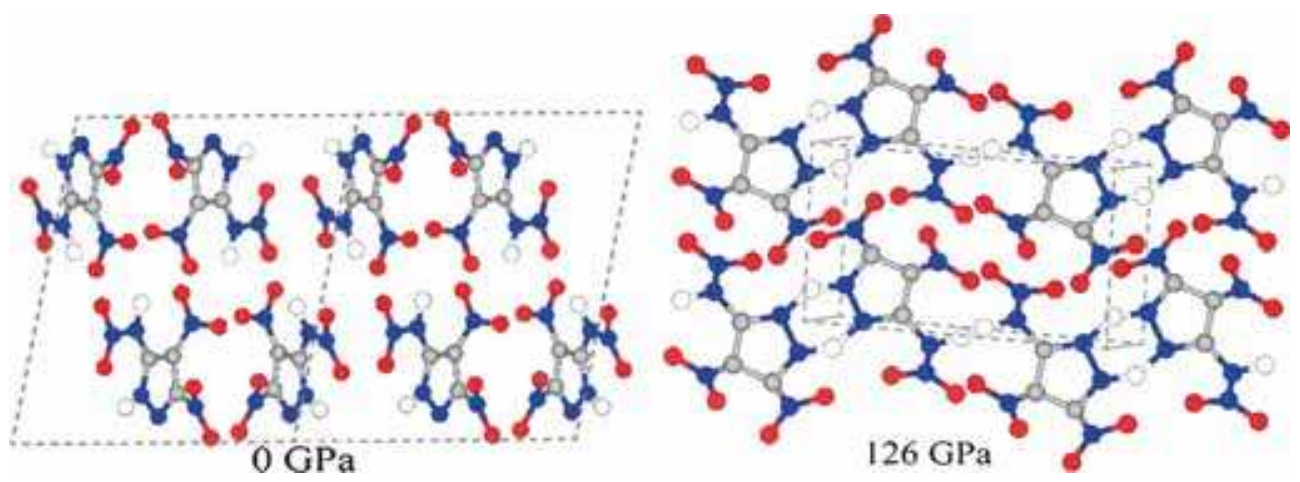

Figure 9. Perspective view of NADNP at $0 \mathrm{GPa}$ and $126 \mathrm{GPa}$. 


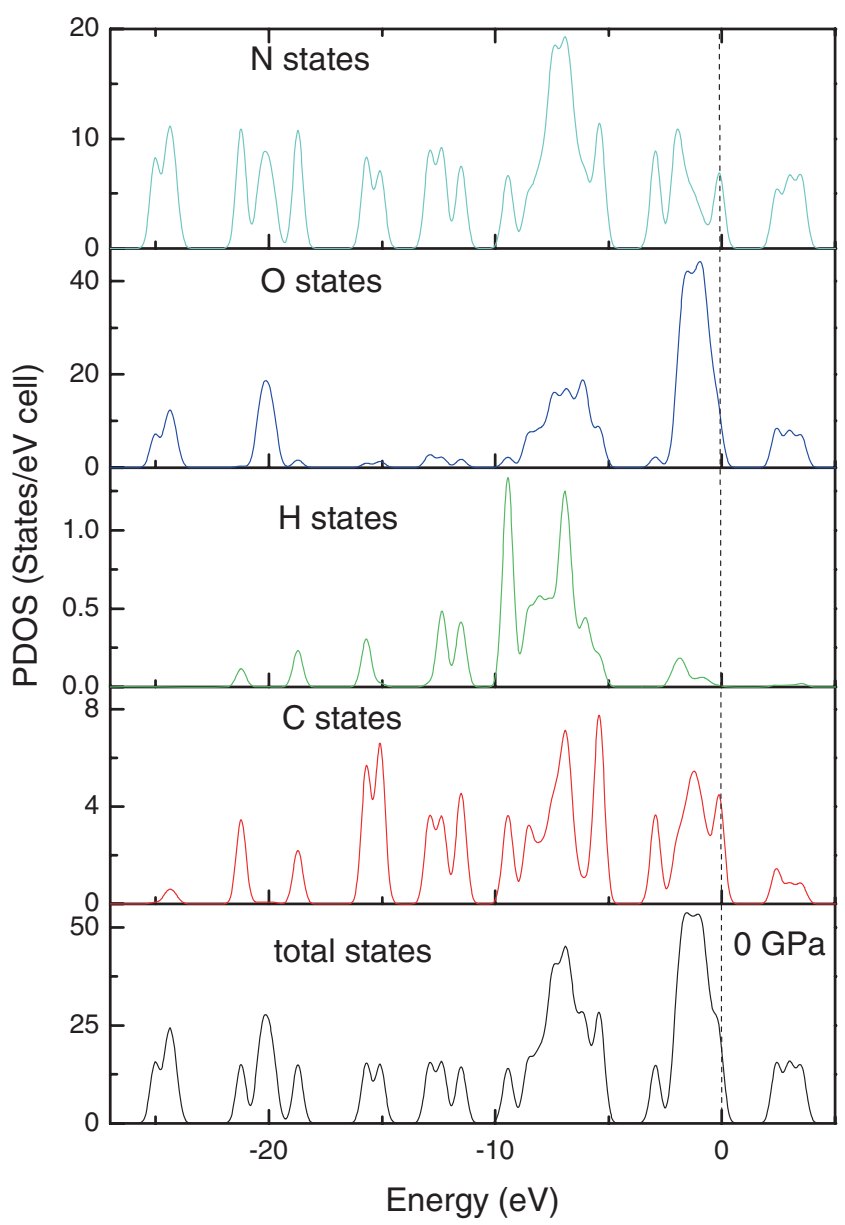

Figure 11. Total and partial density of states (DOS) of the $\mathrm{C}, \mathrm{H}, \mathrm{O}$ and $\mathrm{N}$ states of NADNP at $0 \mathrm{GPa}$. The Fermi energy is shown as a vertical dashed line.

indicating that the electrons have become delocalized completely and can transfer freely between the valence band and conductions band.

Figure 13 displays the PDOS of the N12' and H11 atoms in NADNP at 125 and $126 \mathrm{GPa}$. It is found that there no strong peaks occurred at the same energy in the PDOS of the N12' and H11 atoms at 125 GPa. However, when the pressure increases to $126 \mathrm{GPa}$, almost all the peaks in the PDOS of the N12' and H11 states occur at the same energy, especially in the energy range from -24 to $-6 \mathrm{eV}$, where the shape of the peaks are very alike to each other. In addition, the variation tendency of their PDOS is very similar. For instance, the strong peaks in the PDOS of the N12' atoms occur in -23.7 , $-17.17,-14.87$, and $-1.87 \mathrm{eV}$ at $125 \mathrm{GPa}$, whereas those in the PDOS of the $\mathrm{H} 11$ atoms are relatively weak. The H11 atoms have very strong DOS peaks at -23.44 , $-18.1,-14.55$, and $-9.21 \mathrm{eV}$, and the DOS peaks of the N12' states are also strong. This indicates that the H11 and N12' atoms are bonded strongly at $126 \mathrm{GPa}$.

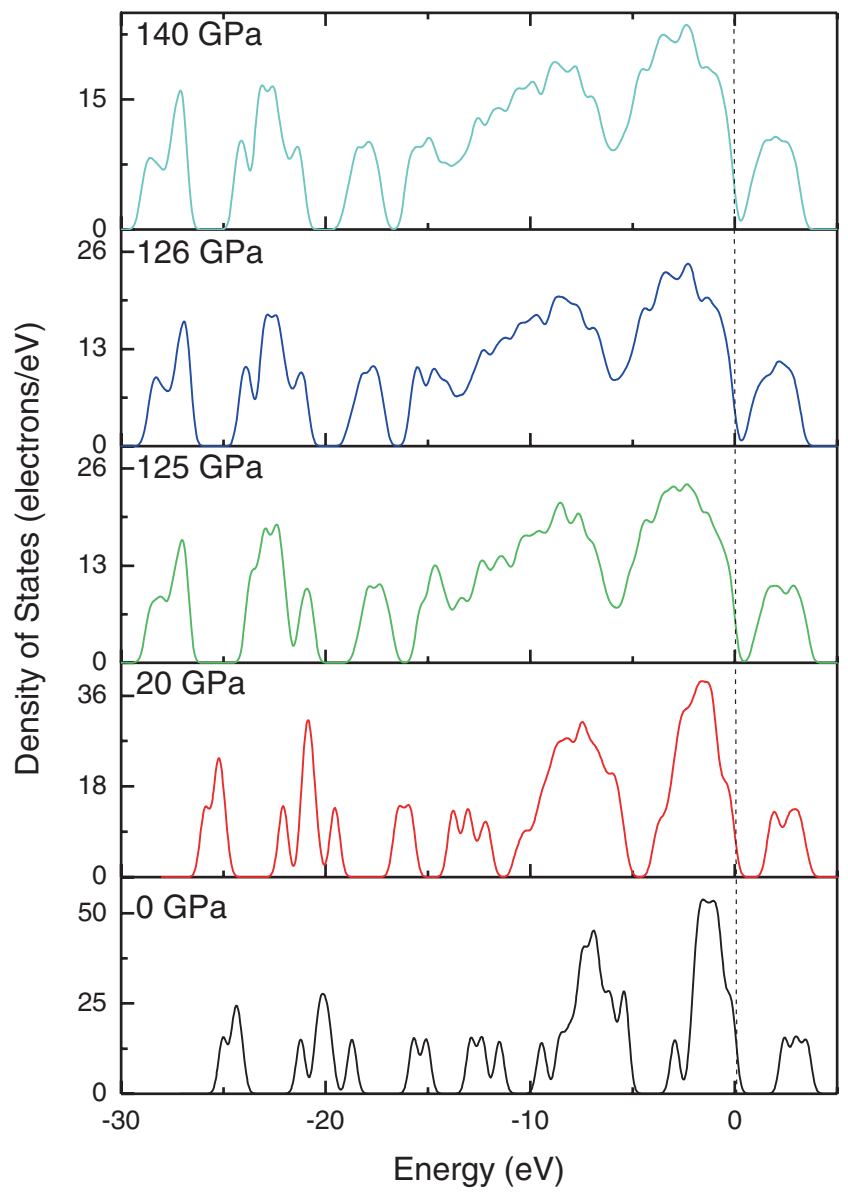

Figure 12. Total DOS of NADNP at the pressure of 0, 20, 125,126 , and $140 \mathrm{GPa}$.

\subsection{Absorption spectra}

In this section, we turn to investigate the optical absorption coefficients of NADNP at different pressures. The interaction of a photon with the electrons in the system can result in transitions between occupied and unoccupied states. The spectra resulting from these excitations can be described as a joint density of states between the valence and conduction bands. The imaginary part $\varepsilon_{2}(\omega)$ of the dielectric function can be obtained from the momentum matrix elements between the occupied and unoccupied wave functions within the selection rules, and the real part $\varepsilon_{1}(\omega)$ of dielectric function can be calculated from imaginary part $\varepsilon_{2}(\omega)$ by KramerKronig relationship. Absorption coefficient $\alpha(\omega)$ can be evaluated from $\varepsilon_{1}(\omega)$ and $\varepsilon_{2}(\omega):^{28}$

$$
\alpha(\omega)=\sqrt{2} \omega\left(\sqrt{\varepsilon_{1}^{2}(\omega)+\varepsilon_{2}^{2}(\omega)}-\varepsilon_{1}(\omega)\right)^{1 / 2}
$$

The absorption coefficients $\alpha(\omega)$ of NADNP at different pressures are plotted in figure 14. The absorption spectra are active over various regions corresponding to the molecular or lattice structures of the individual 


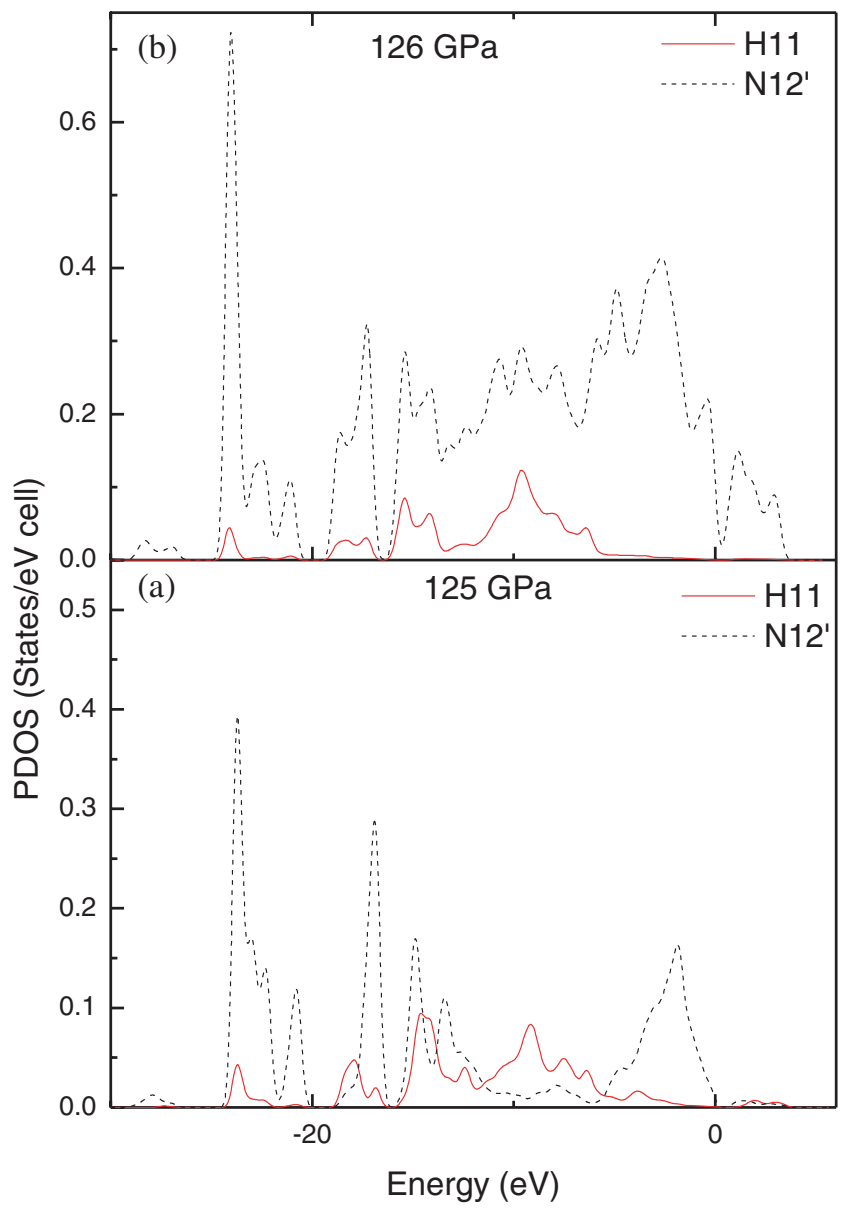

Figure 13. (a) PDOS of the $\mathrm{H} 11$ and N12' atoms at 125 $\mathrm{GPa}$; (b) and at $126 \mathrm{GPa}$.

material. The evolution pattern of absorption spectra for NADNP at different pressures is qualitatively similar. They have an absorption band covering from 0 to 20 $\mathrm{eV}$ and stronger optical absorption from 0 to $8 \mathrm{eV}$. In the energy above $12 \mathrm{eV}$, the absorption coefficients are close to zero. The magnitude of the absorption coefficients of these peaks allows an optical transition due to excitons. At ambient pressure, NADNP exhibits a relatively high absorption coefficient over a relatively few, closely spaced bands. As the pressure increases, the absorption peak of NADNP becomes wider and higher. Upon compression, the DOS peaks in the conduction bands of NADNP have a tendency of shifting to the lower energy. This leads to the decrease of the band gap and so the electron transfer from the valence band to the conduction band becomes easier. Therefore, NADNP has relatively high optical activity at high pressures. However, the absorption spectrum at $126 \mathrm{GPa}$ changes obviously and the absorption coefficients in the energy range of 3.7-11 eV are much more higher than those at $125 \mathrm{GPa}$. This is due to the formation of the H11N12' bond at $126 \mathrm{GPa}$. This also indicates that NADNP

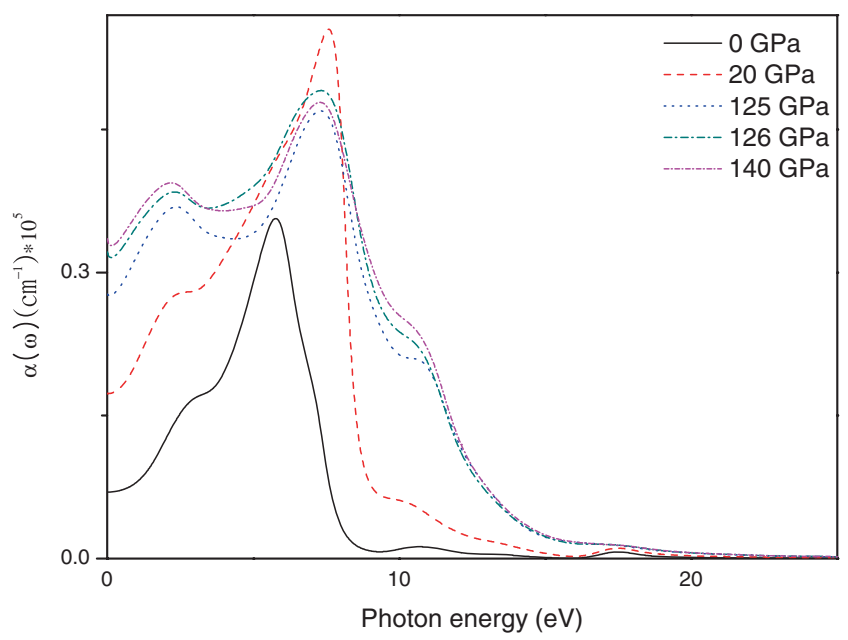

Figure 14. Absorption coefficients $\alpha(\omega)$ of NADNP at different photon energies.

has much higher optical activity at $126 \mathrm{GPa}$ than at 125 $\mathrm{GPa}$. The absorption spectrum at $140 \mathrm{GPa}$ is similar to that at $126 \mathrm{GPa}$, suggesting that the structures at the two pressures are much alike.

\section{Conclusions}

In this work, DFT-D calculations have been performed to study the crystal structure, electronic structure, and absorption properties of crystalline NADNP under hydrostatic pressure of 0-140 GPa. The crystal structure of NADNP was relaxed using three types of vdW corrections such as the PBE-G06, PBE-TS, and PW91-OBS functionals at ambient conditions. The results indicate that the PBE-G06 is the best functional for studying NADNP. At $126 \mathrm{GPa}$, NADNP begins to decompose by forming new H11-N12' bond.

An analysis of the band gap and density of states indicates that NADNP becomes more and more sensitive under compression. Furthermore, the PDOS can provide evidence for formation of new H11-N12' bond at $126 \mathrm{GPa}$. The absorption spectra show that NADNP has relatively high optical activity with the increasing pressure; moreover, the absorption region is broadened in the energy range of above $8.8 \mathrm{eV}$. This work provides useful information in understanding how NADNP behaves under high pressures.

\section{Acknowledgements}

This work was supported by the National Natural Science Foundation of China (Grant No. 21273115) and A Project Funded by the Priority Academic Program Development of Jiangsu Higher Education Institutions. 


\section{References}

1. Yin P, Parrish D A and Shreeve J M $2015 \mathrm{~J}$. Am. Chem. Soc. 1374778

2. Zhu W H, Wei T, Zhang X W and Xiao H M 2009 J. Mol. Struct.: Theochem. 895131

3. Qiu L, Xiao H M, Ju X H and Gong X D 2005 Int. J. Quantum Chem. 148

4. Zhu W H and Xiao H M 2007 J. Solid. State Chem. 180 3521

5. Zhu W H, Xiao J J and Xiao H M 2006 Chem. Phys. Lett. 422117

6. Zhu W H, Xiao J J and Xiao H M 2006 J. Phys. Chem. $B 1109856$

7. Peiris S M, Wong C P and Zerilli F J 2004 J. Chem. Phys. 1208060

8. Zhu W H, Zhang X W, Zhu W and Xiao H M 2008 Phys. Chem. Chem. Phys. 107318

9. Zhu W H, Zhang X W, Zhu W H and Xiao H M 2009 Theor. Chem. Acc. 124179

10. Zhu W H and Xiao H M 2010 Struct. Chem. 21657

11. Wu Q, Zhu W H and Xiao H M 2013 J. Phys. Chem. C 11716830

12. Zhu W H and Xiao H M 2008 J. Comput. Chem. 29176

13. Xiao H M and Ju X H 2004 Int. J. Quantum Chem. 121 12523
14. Fabbiani F P A and Pulham C R 2006 Chem. Soc. Rev. 35932

15. Zhu W H, Xiao J J, Ji G F, Zhao F and Xiao H M 2007 J. Phys. Chem. B 11112715

16. Segall M D, Lindan P J D, Prober M J T, Pickard C J, Hasnip P J, Clark S J and Payne M C 2002 J. Phys: Condens. Matter 142717

17. Payne M C, Teter M P, Allan D C, Arias T A and Joannopoulos J D 1992 Rev. Mod. Phys. 641045

18. Vanderbilt D 1990 Phys. Rev. B 417892

19. Perdew J P and Zunger A 1981 Phys. Rev. B 235048

20. Perdew J P, Burke K and Ernzerhof M 1996 Phys. Rev. Lett. 773865

21. Perdew J P and Wang Y 1992 Phys. Rev. B 4512947

22. Grimme S 2006 J. Comput. Chem. 271787

23. Tkatchenko A and Scheffler M 2009 Phys. Rev. Lett. 102 073005

24. Ortmann F, Bechstedt F and Schmidt W G 2006 Phys. Rev. B 73205101

25. Appalakondaiah $\mathrm{S}$, Vaitheeswaran $\mathrm{G}$ and Lebègue $\mathrm{S}$ 2014 Chem. Phys. Lett. 605-606 10

26. Kresse G and Furthmüller J 1996 Phys. Rev. B 5411169

27. Fletcher R 1980 In Practical Methods of Optimization (New York: Wiley)

28. Saha S, Sinha T P and Mookerjee A 2000 Phys. Rev. B 628828 